\title{
CONCEPCIÓN DEL TRABAJO EN ÁMBITOS PRECARIOS. LOS JÓVENES TRABAJADORES DE MOROLEÓN Y URIANGATO, GUANAJUATO.
}

\author{
Octavio Martín Maza ${ }^{1}$ \\ Miguel Gerardo Pérez Martínez ${ }^{2}$
}

\section{Introducción}

Los resultados presentados en el artículo son producto de la investigación de tesis de posgrado, la cual parte de la pregunta; ¿por qué los jóvenes insertos en el mercado de trabajo precario vislumbran permanecer en él, al orientar sus aspiraciones laborales dentro de este mismo mercado? La hipótesis que orientó la investigación se refiere a que el joven de la zona metropolitana de Moroleón y Uriangato, Gto. que trabaja en las tiendas de ropa, desarrolla sus aspiraciones en el mercado de trabajo precario, ya que es en este contexto laboral en el que ha construido una representación social con el sentido del trabajo, además de que en este contexto obtiene su capital social. Los resultados que se presentan son producto de una encuesta y de acercamientos etnográficos al campo.

La zona metropolitana compuesta por Moroleón y Uriangato, es una conurbación intermunicipal $^{3}$ (mapa 1). El municipio de Moroleón cuenta con una población total de 50,377, por su parte Uriangato concentra una población total de 62,761, sumando en conjunto 113,138 (INEGI, 2015). Específicamente, Uriangato se ubica al sur del estado de Guanajuato, el cual colinda al norte al este con el municipio de Yuriria y al oeste con

\footnotetext{
${ }^{1}$ Universidad Autónoma de Aguascalientes, México. Email: octaviomazadc@gmail.com ORCID id: https://orcid.org/0000-0002-3991-7751

${ }^{2}$ Instituto para las Mujeres Guanajuatenses, México. Email: imug@guanajuato.gob.mx ORCID id: https://orcid.org/0000-0002-1972-7272

${ }^{3}$ El término refiere "al conjunto de dos o más municipios donde se localiza una ciudad de más de 50 mil o más habitantes, cuya área urbana, funciones y actividades rebasan el límite del municipio que originalmente la contenía, incorporando como parte de sí misma o de su área de influencia directa a municipios vecinos, predominantemente urbanos, con los que mantiene un alto grado de integración socioeconómica" (Maza y Ortíz, 2015: 21)
}

Iluminuras, Porto Alegre, v. 21, n. 52, p. 23-49, abril, 2020. 
Moroleón, al sur con Cuitzeo y Santa Ana Maya, ambos pertenencientes al estado de Michoacán (Maza y Ortíz 2015). Estos municipios, que se caracterizan en lo económico, por la confección de ropa de dama, de algodón y prendas de vestir de tejido de punto, son reconocidos nacionalmente como motores importantes dentro de la industria de la confección estructurado a partir de pequeños talleres (Arias, 1992).

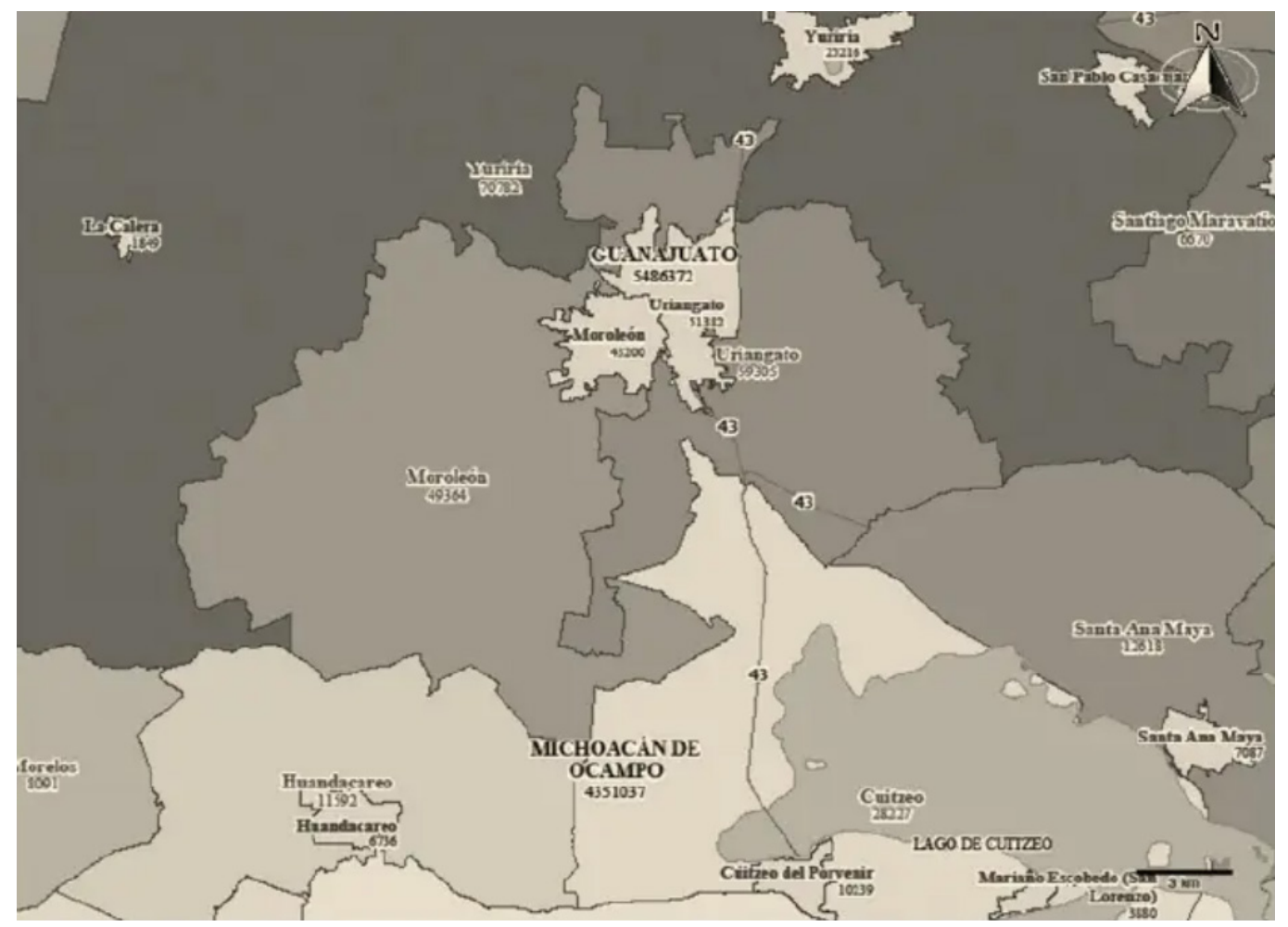

Fuente: retomado de Maza y Ortíz (2015).

La informalidad y la precariedad laboral surgen como fenómeno de interés a finales del siglo $\mathrm{XX}$, aunque su presencia en la vida cotidiana del trabajador latinoamericano y particularmente del mexicano tiene una larga historia (Negrete, 2011; Reygadas, 2011, Salas, 2006 y Tokman, 2011). Desde que el concepto (de informalidad) empezó a usarse con los estudios realizados por la Organización Internacional del Trabajo sobre las condiciones de trabajo en Ghana y Kenia en 1971 y 1972, se pudo observar que, en los 
países en vías de desarrollo, los problemas laborales no se reducían al desempleo, sino que a la par de éste se daban una serie de fenómenos relacionados con las condiciones en que se producían los bienes y servicios (Negrete, 2011).

De manera específica los estudios sobre la forma en la que los jóvenes se integran al mercado de trabajo, nos remiten a los estudios de las desigualdades, en este sentido Saraví (2015) realiza una investigación sobre su constituciónye y plantea la necesidad de analizar la forma en la que los procesos macrosociales se corporizan en las biografías. La forma en la que los jóvenes se acercan al mercado de trabajo tiene que ver con la experiencia y con la articulación de prácticas y sentidos, mismo que no se agota sistemas clasificatorios. Se construye además como lo indica Reygadas (2008) en los diferentes campos, especialmente en el mercado y el estado; siendo el primero donde se premia, tanto la eficiencia, como la productividad y donde se hacen evidentes las diferencias; las cuales, finalmente, constituyen al capitalismo contemporáneo.

Acorde con la pregunta de Reygadas (2008) nos interesa ver la forma en la que los actores combaten las desigualdades; es decir que dentro del enfoque multidimensional que plantea, rescatamos lo microsocial, que atiende a los recursos y capacidades de los individuos. Se debe aclarar que no estamos negando los otros aspectos (la dimensión macro) que el autor menciona, sino tratando de explicar la forma en la que los sujetos justifican su permanencia en ciertos espacios laborales, los definen y los defienden. Atendemos a la forma en la que este proceso es legitimado y construido por los sujetos, además de los mecanismos de compensación que se construyen en cada espacio particular. Por lo que, consideramos pertinente indagar sobre los significados que los jóvenes tienen sobre trabajo que realizan y las razones que convierten esa actividad, en algo en lo que vale la pena permanecer.

En el trabajo realizado por los vendedores de ropa en la Zona Metropolitana Moroleón-Uriangato predominan las condiciones de informalidad (tal como se explica más adelante), lo que supondría valoraciones negativas, sin embargo, los jóvenes encuentran ventajas que dan sentido a estas actividades. Las categorías de informalidad y precariedad sirvieron como referente para comprender el mundo del trabajo en particular el de los 
jóvenes; por otra parte, la teoría de las representaciones sociales permitió la articulación entre las categorías mencionadas y fungió como enfoque metodológico para obtener y analizar los resultados de la investigación.

En consonancia con lo arriba mencionado, este artículo tiene como objetivo explicar los significados que los jóvenes que laboran en las tiendas de ropa tienen sobre el trabajo. En la primera parte del artículo se describen aspectos demográficos, territoriales y los relacionados con el proceso histórico que la industria textil y del vestido ha experimentado en la región analizada. En la segunda parte se discuten los conceptos de informalidad y precariedad laboral, en particular en el caso de los jóvenes, así como de las aportaciones del concepto de representaciones sociales. En tercer lugar, se muestra el apartado metodológico, en el que aborda tanto el método, como las técnicas utilizadas para la recolección de datos. En la cuarta parte se presentan los resultados obtenidos en relación con las condiciones de trabajo y los significados que los jóvenes han generado de su actividad laboral.

\section{La construcción de un mercado de trabajo}

Como resultado de la fabricación de prendas de vestir, los productores se vieron en la necesidad de constituir un mercado específico para su comercialización, así que durante la década de 1950 se estableció el mercado de ropa de Moroleón, el cual se vio favorecido por la mejora en las carreteras. Para la década de 1960, el mercado se encontraba en mejores condiciones y su entorno se caracterizaba por un desarrollo urbano notable, en la década de 1970 se caracterizó por la introducción de nuevas materias primas, como la lana, el algodón y el acrilan, que produjeron un abaratamiento en la producción de mercancías y para la década de 1980 en Moroleón había más de 1000 empresas, 80\% constituido en talleres familiares y $20 \%$ de naturaleza mayor, con una cantidad mínima de 15 trabajadores, mientras que en Uriangato se calculaban 100 talleres organizados a nivel familiar (Sierra, 2003). A la par de este fenómeno, la comercialización de ropa en la ciudad seguía creciendo y el mercado que se había constituido en la década de 1950 tomaba más fuerza durante la década de 1980. Sierra (2003) señala que la vasta producción de prendas de vestir consolidó la venta en ambos municipios, dando pie a la apertura de locales y tianguis 
especializados en la venta de ropa. En 2018, Moroleón y Uriangato contaban en su totalidad con 2,039 unidades económicas reconocidas como comercios al por menor de ropa, bisutería y accesorios de vestir, de las cuales 1,180 estaban en Uriangato y 859 en Moroleón de acuerdo con el DENUE (2018).

En este mercado laboral se ocupan jóvenes, en su mayoría mujeres, quienes de acuerdo con lo observado por García (2015) resultan ser personas con bajo nivel educativo y redes sociales muy débiles ${ }^{4}$. En este sentido llama la atención que la revisión de las condiciones de trabajo refleja que la mayor vulnerabilidad se encuentra en las personas que trabajan en el comercio, ya que se trata de la actividad que más fácil acceso, al exigir poca experiencia laboral. En términos generales el trabajo en el comercio implica atender al cliente, es decir mostrar los distintos diseños y tallas para comercializar al menudeo o al mayoreo, además de la organización de la mercancía e inventarios cuando estos son requeridos. Como se puede observar, el sector comercio en ambos municipios es una importante fuente de ocupación, sólo superada por la industria manufacturera, la cual es el motor de la región y el mercado laboral que ofrece los mejores salarios. Esto se explica por la forma en la que se ha configurado la organización del modelo de producción y, además, permite comprender la sobrevivencia de esta forma de hacer negocios; en particular nos referimos al predominio de los pequeños talleres, con un alto grado de flexibilidad.

\section{La condición del trabajo y su relación con las representaciones sociales}

Un concepto que articula lo debatido en este artículo es el trabajo, mismo que Pérez Islas y Urteaga definen como: "una construcción social, referida a un contexto histórico y cultural específico; así como a las experiencias y relaciones simbólicas que los individuos

\footnotetext{
${ }^{4}$ García (2015) realizó una medición de las redes sociales de trabajadores de la zona en estudio. Basándose en el análisis del capital social y el prestigio ocupacional menciona que en “...los empleados de mostrador, el contexto es diferente. Se trata de personas muy jóvenes, con estudios medios o de bachillerato y muy activas socialmente. Los datos parecen indicar, sin embargo, que esta actividad social tiene características que afectan su acceso potencial a recursos que... pueden significar beneficios instrumentales, como por ejemplo conseguir un mejor trabajo" (: 139). En términos generales la debilidad referida se entiende como un deficit relacional, ya que están menos conectados que quienes laboran en campos de la producción.
} 
establecen con un modo de vida determinada" (Pérez Islas y Urteaga, 2001: 360), a lo que se debe añadir que tiene una profunda incidencia en la vida privada de los trabajadores.

Las formas de contratación que se enmarcan dentro de la informalidad, con la ausencia de contratos claramente definidos y de prestaciones legales, permiten justificar por qué se toma como punto de partida la informalidad para el análisis de este modelo de producción. Las unidades productivas actúan en búsqueda de ganancia y, tal como anota, Tokman (2011) la finalidad de los trabajos informales es generar mayor ganancia para las empresas. La estrategia de ganancia se da a partir de dos condiciones de la informalidad, la primera ocurre con la descentralización del proceso de producción y la segunda con la subcontratación de mano de obra.

En este sentido se atribuye a la informalidad un doble papel: por un lado, el de la reducción de costos $\mathrm{y}$, por otro, el de la degradación del poder de los trabajadores, expresado en la reducción de su capacidad de organización y defensa. Ambas vías concurren en la afectación y desprotección de los trabajadores, pues la tarea de bajar los costos de producción está diseñada, entre otras cosas, para reducir las responsabilidades de la empresa, es decir: la intención de las empresas, al hacerlo por la vía informal, es no entablar ninguna relación laboral que las obligue a tener responsabilidades legales para con el trabajador (Maza y Gutiérrez, 2015). En el caso mexicano, la informalidad es una de las principales formas para establecer relaciones de trabajo, ya que representa el $58.1 \%$ de la población (ENOE, 2014) y dadas sus características hace proclive la aparición de precariedad al establecerse relaciones laborales sin la existencia de contratos de trabajo y sin protección social. Si bien, las primeras aproximaciones teóricas se establecieron en la década de los 80, Maza (2008), Rojas y Salas (2011), Mora (2011) y Reygadas (2011) advierten que el debate no está concluido puesto que siguen apareciendo y aumentando algunas características enmarcadas en el concepto de precariedad: inestabilidad del empleo, desprotección social y salarial, el incumplimiento de los derechos laborales y las deficiencias en la seguridad social; mismas que como veremos adelante son parte constituyente del trabajo de los jóvenes. Al respecto Reygadas (2011) señala que:

En el marco de la globalización los procesos de ajuste estructural y la ruptura de muchos de los pactos laborales que se presentaron en la época del Estado de Bienestar, han proliferado los 
trabajos precarios, es decir, empleos inestables, sin contrato, con salarios bajos, sin prestaciones, con jornadas irregulares, a tiempo parcial o demasiado largas, con malas condiciones de trabajo, carentes de seguridad social, violatorios de los derechos laborales, con nula o reducida negociación colectiva (Reygadas, 2011: 22).

La desprotección laboral se encuentra relacionada con la omisión de los derechos laborales, lo que significa que los sujetos que se desenvuelven laboralmente bajo esta condición disponen de escaso poder de control sobre las condiciones en las que operan, lo que trae consigo múltiples implicaciones que rebasan la dimensión económica, pues se ven ignorados otros componentes como la salud del trabajador, el acceso al sistema de seguridad social, la duración de la jornada estipulada por la ley, la remuneración del tiempo extra, la realización de sus actividades en condiciones dignas que le provean de respeto y de seguridad al momento de ejercerlos, así como en el acceso a un sistema de pensiones para el retiro. La inestabilidad laboral refiere a la condición en la cual el trabajador, dada la relación contractual que ha establecido, puede perder su trabajo en cualquier momento, sin que por ello el patrón esté comprometido a indemnizarlo, generando reducida temporalidad de los trabajos, condición que trae consigo incertidumbre(s) y, por tanto, falta de previsibilidad respecto al futuro.

La mitad de los trabajadores en México no cuentan con el derecho a servicios de salud o de pensión emanados de su condición de trabajadores Rojas y Salas (2011). Por su parte, la situación de los salarios en México se ha caracterizado por su relación con la insuficiencia para satisfacer las necesidades básicas de su población (Reygadas, 2011, Rojas y Salas, 2011). Sin embargo, esta cuestión se ha agudizado a partir de la década de 1980 por los constantes estados de crisis económica en los años de 1982, 1994 y 2008 y que al parecer se ha convertido en una situación constante hasta la fecha (Zapata, 2005). Sumado a esta serie de hechos, la economía globalizada ha traído consigo un modelo de competitividad entre numerosos países para adquirir inversiones de capital extranjero con el propósito de reactivar la economía interna. Esta competitividad se ha llevado a cabo en escenarios técnicos, administrativos y jurídicos, regulados dentro de un marco económicopolítico que ha venido a legitimar la precariedad del trabajo y que, en consonancia con esta lógica, se presenta como uno de los principales incentivos para la inversión la mano de obra barata. 
El panorama del mercado de trabajo general requiere algunos matices cuando abordamos el trabajo de los jóvenes, es un tema que ha sido investigado por diversos autores dando lugar a reflexiones que contribuyen a la compresión del problema que analizamos aquí. De manera específica se ha puesto atención en la forma en la que se accede al mundo del trabajo, donde el paso de la escuela al mundo laboral es fundamental. Saraví (2009) caracteriza el trabajo de los jóvenes como inestable y desprotegido, oscilando entre la precariedad y el desempleo a lo que se debe añadir la acumulación de desventajas de los jóvenes desfavorecidos. A lo que se debe añadir la condición determinante que ejerce el origen, en particular el nivel de estudios de las madres, quienes son las que transmiten las desventajas o las ventajas.

En este sentido Pérez Islas y Urteaga (2001) abordan la problemática de la forma de obtener un trabajo, dónde buscarlo y cómo, a lo que añaden las múltiples contradicciones que este proceso implica. Resulta relevante observar que, en espacios diversos, como el que estudiamos con un modelo de producción basado en los pequeños talleres, con predominio de contratos informales, se requiere comprender la forma en la que los sujetos se incorporan al trabajo y cuáles son las compensaciones (Reygadas, 2008) que perciben. Se trata de un modelo que ha configurado un funcionamiento en el que como lo mencionan los estudios clásicos de los distritos industriales el negocio está en el aire y, por lo tanto, el acceso al mercado laboral no parece ser un problema, sino más bien las razones para permanecer en él. En el mismo sentido encontramos que la estabilidad de los puestos de trabajo depende de condiciones tales como la capacidad de hacerse indispensables, lo que también se logra mediante el fortalecimiento de las relaciones con sus patrones, tal como lo documentan Pérez Islas y Urteaga (2001).

De acuerdo a las observaciones y entrevistas realizadas a lo largo del proceso de la investigación, trabajar en los talleres y las tiendas representa mantener lazos informales entre trabajadores y patrones, en los cuales las jornadas laborales no son estables, por el contrario, están caracterizadas por la temporalidad del trabajo, la cual está determinada por las circunstancias (y diseños) de la moda global (Pasillas, 2016). Una característica importante en este modelo de producción de los pequeños talleres es que el trabajo mantiene una relación muy cercana con la familia, en tanto que el apoyo mutuo es una 
forma cotidiana de hacer negocio, también la confianza es una condición que fortalece los vínculos entre trabajadores y patrones, la producción, comercialización y todos los componentes propios del trabajo en el lugar

El trabajo ideal se construye a partir de las propias condiciones del mercado de trabajo, en el caso que nos ocupa el ideal positivo se genera a partir de las posibilidades que da la propia condición en la que se trabaja. De la misma manera sucede la construcción de las certidumbres, es decir en estos casos las garantías de permanencia no las otorga un contrato, sino las estrategias de los propios trabajadores (Pérez Islas y Urteaga, 2001).

En las líneas siguientes presentamos una breve revisión sobre las representaciones sociales, mediante las cuales analizamos el contexto en donde se desenvuelven laboralmente los jóvenes; así como, los mecanismos que les permiten dar sentido y significado al trabajo mediado por las condiciones de precariedad laboral.

Las representaciones sociales permiten interpretar y pensar la realidad cotidiana, brindan una forma de conocimiento social que se constituye de múltiples maneras: a través del contexto concreto en que se sitúan los individuos y los grupos, de la comunicación que se establece entre ellos, de los aspectos culturales, los códigos, valores e ideologías relacionados con las posiciones sociales específicas (Moscovici, 1988; Jodelet, 1988 \& Abric, 2001). Tal como se menciona en Jodelet (1988):

El concepto de representación social designa una forma de conocimiento específico, el saber de sentido común, cuyos contenidos manifiestan la operación de procesos generativos y funcionales socialmente caracterizados. En sentido más amplio, designa una forma de pensamiento social (p. 474).

Las representaciones sociales se componen por un conocimiento construido socialmente, mismo que provee al sujeto de elementos para entender la realidad y dar sentido a sus acciones. De igual forma manifiesta que dicho conocimiento está formado de significados, ideas y percepciones que dan sustento a la visión de mundo, la cual es desarrollada por el sujeto acorde con su contexto social, económico e ideológico más próximo y a su historia personal (Abric, 2001). 
Si bien, las representaciones sociales aluden tanto a un aspecto cognitivo como a un aspecto social, es en este último en el que nos centraremos, dado el interés que guía a este estudio. Las representaciones sociales conciernen a lo que Jodelet (1988) denomina conocimiento espontáneo, ingenuo, o, en otras palabras, conocimiento de sentido común. Ésta es construida a partir de la experiencia y el contexto social más próximo en el que se desenvuelven de manera cotidiana los individuos. Lo anterior muestra la relevancia de entender la forma en la que los jóvenes construyen sus sentidos del trabajo en un contexto específico y, la manera en la que construyen sus planes a futuro.

Algunas características de los jóvenes en estos lugares son las siguientes; en su mayoría viven con sus familiares, ya que como se mencionó, el vínculo familiar es central para comprender otras dinámicas sociales y laborales. También, gran parte de los jóvenes que laboran en los municipios viven allí mismo, no obstante, otro contingente proviene de municipios aledaños, los cuales principalmente están insertos en los talleres y comercios con la figura de empleado y no comparten rasgos familiares con sus empleadores. Otro grupo de jóvenes son los hijos de empresarios y/o dueños de talleres, los cuales están más encaminados al estudio universitario de diversas ramas de la producción, y que poco a poco se van sumando a las actividades del negocio familiar y van implementando algunas estrategias aprendidas en la escuela. Finalmente, las redes sociales y de amistad son de suma relevancia para la inserción de los jóvenes al mercado de trabajo

\section{Estrategia metodológica}

El estudio se llevó a cabo en el mercado de ropa de las ciudades de Moroleón y Uriangato. Los locales y puestos de ropa se encuentran establecidos en una serie de calles continuas que inician en Uriangato y que terminan prácticamente en el centro de la ciudad de Moroleón. Mediante un muestreo por conveniencia, se aplicaron 104 cuestionarios y el mismo número de cartas asociativas, que estuvieron divididos en 76 mujeres y 28 hombres que se encontraron laborando, como vendedoras(es) en 83 tiendas de ropa. De entre estos 104 encuestados, se realizaron 10 entrevistas a jóvenes que mostraron interés particular en 


\section{Concepción del Trabajo en Ámbitos Precarios. Los jóvenes trabajadores...}

la investigación. El estudio se llevó a cabo con jóvenes de entre 15 y 29 años, con ingresos ${ }^{5}$ diarios que oscilan entre los 80 y 120 pesos mexicanos.

La forma en que se accedió a ellos fue de manera directa acudiendo a las tiendas de ropa y una vez que se les explicó el motivo de la visita, se les solicitó llenar de manera individual un cuestionario y realizar, en conjunto con el entrevistador, una carta asociativa. A quiénes mostraron disposición se les solicitó una entrevista. Cabe mencionar que, atendiendo a las condiciones de trabajo, las entrevistas se realizaron en lapsos de descanso, o de poca afluencia de clientes en el horario de trabajo.

El cuestionario aplicado está integrado por tres constructos teóricos: datos generales, trayectoria laboral y sobre las condiciones de trabajo. Por su parte, las entrevistas semi-estructuradas fueron realizadas a 6 mujeres y 4 hombres e incluyó tres ejes temáticos: trayectorias laborales, condiciones de trabajo y aspiraciones laborales. En relación con la técnica de la carta asociativa, se trata de un método de asociación libre, fundado en trabajo verbal que permite constreñir el límite de la expresión del sujeto. La dinámica que se usó para aplicar esta técnica fue la de introducir al joven en el ejercicio, mediante un término inductor, que fue el término "trabajo", y a partir del cual se le pid mencionar cuatro palabras asociadas con el término inductor, para posteriormente emitir tres nuevas palabras tomando como base la primera y una de las segundas, hasta completar el ejercicio.

La meta al aplicar esta técnica era que la cadena de palabras asociadas a partir del término inductor, generan un reflejo de la representación social que ha construido el sujeto a partir del sentido que tiene para él el trabajo (Abric, 2001). Una vez que se registraron y analizaron las palabras emitidas por los jóvenes, se procedió a construir categorías que ayudarán a conglomerar el sentido que cada una de ellas representaba. La estrategia metodológica se complementa con acercamientos a archivos, documentos e investigaciones ya realizadas, así como a censos y bases de datos oficiales que ayudaron con la obtención

\footnotetext{
${ }^{5}$ De acuerdo al Banco de México, el tipo de cambio es de $\$ 19.77$ pesos mexicanos por dólar. Siguiendo este caso de estudio, el ingreso promedio de los trabajadores oscila hasta en 6 dólares por día. Esto representa un promedio de 36 dólares por semana. considerando que se descansa 1 día por semana. Retomado de https://www.banxico.org.mx/. Consultado el 4 de marzo de 2020.
} 
de datos que contribuyeron a la comprensión del fenómeno. Los jóvenes que participaron en el estudio se encuadran en el siguiente perfil.

- Edad entre 15 y 29 años.

- Refieren mayoritariamente a niveles de estudio de secundaria y preparatoria. El $51.92 \%$ cuentan con secundaria; el $42.30 \%$ cuentan con preparatoria y el $5.76 \%$ tiene el nivel de primaria.

- En promedio cuentan con una trayectoria laboral de casi 4 años.

- Su salario por día es entre los 80 y los 120 pesos mexicanos (aproximadamente entre 4 y 6 dólares américanos).

- No cuentan con ningún tipo de prestación social, la única recompensa que obtienen por su trabajo es su sueldo semanal.

\section{Situación del trabajo y sus significados}

En lo que respecta a la trayectoria laboral, los jóvenes que participaron en este estudio tienen en promedio una vida laboral de 3.9 años, lo que se traduce en un inicio aproximado a los 16.7 años y, con una media de duración de su primer empleo de 15 meses, mientras que en el empleo con el cual contaban al momento de la encuesta el 57\% de los jóvenes llevaban menos de 1 año.

El $80 \%$ de los participantes ha comenzado su vida laboral en el sector servicios, mientras que el 20\% se inició en talleres de confección de ropa. De este total, el 38\% ha dejado su trabajo en tiendas de ropa al menos una vez para trabajar en talleres o fábricas de ropa, sin embargo, han regresado a las tiendas. Cabe aclarar que, de acuerdo a lo observado en investigaciones previas, los espacios que ofrecen mejores condiciones de trabajo son los talleres de confección.

Por su parte, el $60 \%$ de los jóvenes tienen como principal motivo para entrar a laborar el hecho de ayudar en los gastos familiares, seguido de solventar gastos personales con el 26\%, desarrollo personal con el 8\%, solventar gastos de la escuela con el 5\% y, finalmente, en la opción otro, se da el 1\%, que refiere a un joven que tenía como principal motivo juntar dinero para poner un negocio propio. Estos datos nos permiten reflexionar 
con Saraví (2009) sobre las condiciones para ingresar al mercado de trabajo, ya que al afirmar que se tenía que trabajar, se aducen necesidades que resultan incompatibles con el estudio. Lo que termina por convertir al trabajo "en una fuente de ingreso para gastos personales y como fuente de identidad y autonomía" (Saraví, 2009: 94). Además, como se observa en los fragmentos presentados a continuación, se debe rescatar la figura de la madre como factor determinante de la trayectoria. Un número considerable de jóvenes encuestados manifiestan ${ }^{6}$ que su inserción en el trabajo parte de la necesidad económica de solventar necesidades básicas ${ }^{7}$ :

Cuando entré a trabajar estaba chiquilla (pequeña), me acuerdo que me dio trabajo la señora a quien mi mamá le ayudaba con el quehacer de la casa, en ese entonces la situación en mi casa no era muy buena, bueno ahora tampoco, pero si me acuerdo que batallábamos mucho porque no había mucho dinero, bueno ahora tampoco, le digo, ijaja! pero si trabajábamos para comer, me acuerdo que la primera paga que me dieron se la di entera a mi mamá... (Conversación personal, mujer de 24 años, 2015).

Cuando entré a trabajar lo hice porque me quería comprar ropa y un celular, y bueno si, de vez en cuando me doy mis lujillos (lujos), pero pues está bien difícil no creas, viendo cómo está la situación en la casa pues mejor decido ayudar en la casa, porque se necesita, entonces tengo que dar el chivo (alimentación), y no me pesa eh, porque sé que es para la comida o para pagar el agua o la luz, bueno cosas que se necesitan... (Conversación personal, hombre de 21 años, 2015).

En lo que concierne a las condiciones de trabajo resultó que el $100 \%$ de los participantes carecen de contrato laboral por escrito. $\mathrm{Y}$ en relación con la pregunta ¿qué prestaciones recibe? 89\% afirmó contar, adicional a su sueldo, con aguinaldo; de tal manera que otras prestaciones como reparto de utilidades, créditos de vivienda, guardería, fondo de retiro o seguro social (IMSS) nunca fueron señaladas.

Una de las problemáticas que define de mejor manera la informalidad es la carencia de servicios de seguridad social. Por lo que se inquirió, de manera expresa, sobre la manera en la que se solucionan los problemas de salud al no contar con seguro social. Los entrevistados respondieron que existen alternativas para atender esas situaciones, tales como el seguro popular, las farmacias similares, médicos privados, remedios caseros, o bien, cuando la situación era grave, la venta de bienes para solventar los gastos. No

\footnotetext{
${ }^{6}$ Se retoma la postura de James Clifford (1988) en tanto que se busca representar adecuadamente la autoridad de los informantes.

7 Se incluye en paréntesis sinónimos para facilitar la comprensión del habla popular para el lector internacional.
} 
obstante, otra respuesta que se generaba al respecto, por parte de las y los entrevistados era que su condición de juventud no les permitía pensar en la salud como una necesidad importante, ya que aún se tenía la fuerza para realizar el trabajo durante la jornada laboral. Tal vez esta es una de las condiciones que dejan más en claro la condición de vulnerabilidad que representa el trabajo de estos jóvenes:

...la verdad eso no me preocupa porque cuando se trata de una gripa, ps ya ves que está el simi (médicos de farmacia), aunque también tengo seguro popular pero la verdad no me gusta ir porque hay mucha gente y luego ahí atienden bien feo (mal), no me gusta, lo bueno es que casi no me enfermo, es lo bueno de estar joven y bella, jajaja! por lo mismo no me fijo tanto en eso, si me dan o no seguro, como que no me fijo, aparte como te digo nadie da seguro aquí... (conversación personal, mujer de 19 años, 2015).

La condición de precariedad se expresa en la idea de que sólo se requiere solucionar lo inmediato, sin tener proyectos a futuro o sin considerar posibilidades como la de tener una enfermedad a pesar de la juventud. Esta situación concuerda con los datos obtenidos, en los que observamos que, no resulta relevante el cambio de trabajo para obtener mejores prestaciones sociales, situación que hace evidente la precariedad laboral como una condición normalizada. Además, que nos permite plantear un problema fundamental, en el que se prefieren los mejores ingresos en vez de los contratos formalizados, los cuales tienden presentar salarios más bajos.

En lo que se refiere a las remuneraciones, el promedio de ingreso mensual de un trabajador de las tiendas de ropa de Moroleón y Uriangato es de 3102 pesos (157 dolares americanos). Si este salario se compara con el percibido por trabajadores en otros rubros, se encuentra que por arriba de los recibidos por empleados de sección en las tiendas de autoservicio (gráfica 1).

Gráfica 1: Promedio de ingresos mensuales en diversas actividades (pesos mexicanos). 


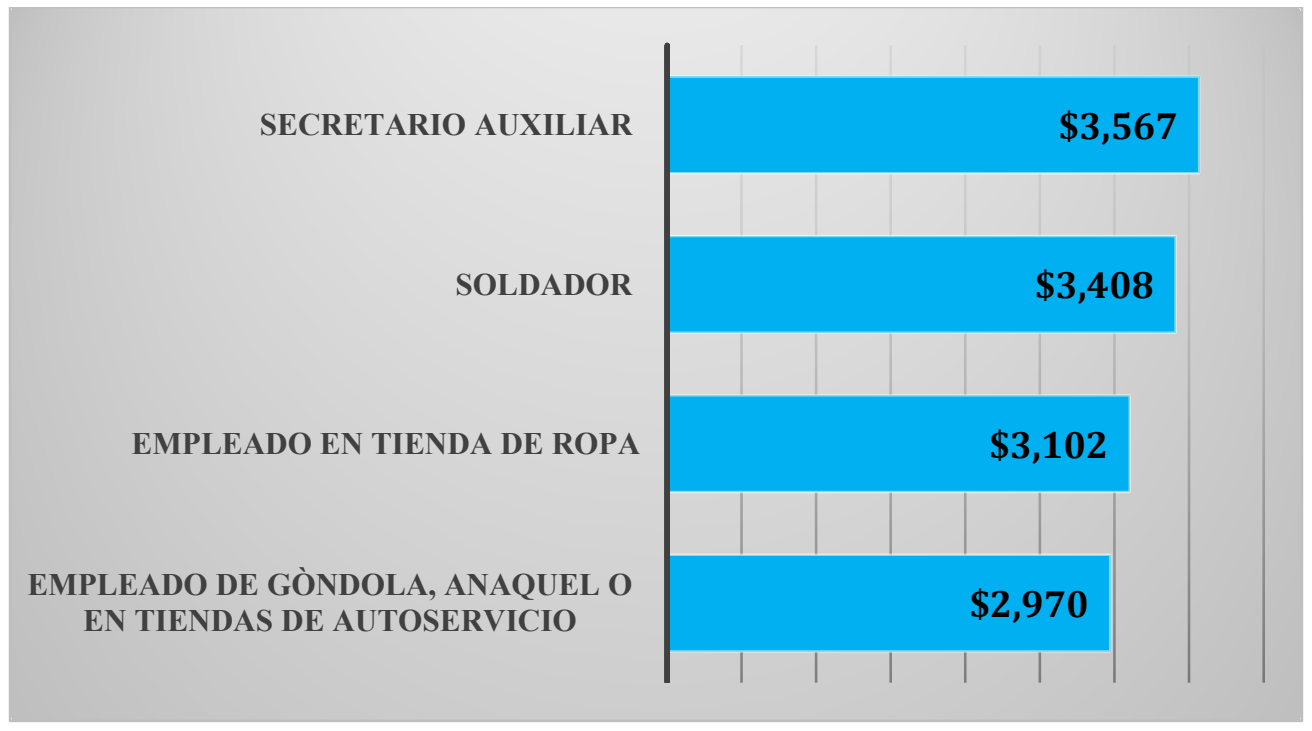

Fuente: Elaboración propia con datos de Comisión Nacional de los Salarios Mínimos 2018 y Cuestionario aplicado a jóvenes participantes en el estudio (STPS).

Este comparativo deja ver que, aunque los sueldos son bajos, en relación, a las horas trabajadas, resultan competitivos ante otras alternativas de trabajo que implican la condición de ser empleado y de no ser profesionista. En relación con las horas de trabajo, el promedio de horas laborales por semana asciende a 50.6 horas. El mínimo de horas laboradas semanalmente fue de 19, mientras que el máximo fue de 69 por semana.

Por otro lado, en lo que concierne a los significados del trabajo construidos por los jóvenes, se podría pensar que en un contexto laboral como el de Moroleón-Uriangato, donde la falta de garantías laborales se presenta como imperiosa, la percepción negativa del trabajo y el no aprecio por el mismo dadas las circunstancias en las que se desempeña estaría muy presente; sin embargo, los resultados encontrados en este estudio muestran otras dimensiones relevantes para la comprensión de las acciones llevadas a cabo por los jóvenes en relación a su vida laboral. Hasta aquí hemos hablado de los datos que reflejan las condiciones de trabajo, en lo que sigue buscaremos aportar datos sobre las representaciones sociales sobre el trabajo.

A partir del análisis de representaciones sociales del trabajo, se analizó: primero la valoración positiva o negativa del trabajo; $\mathrm{y}$, segundo, los significados del trabajo: 
- $66 \%$ de los encuestados manifestó tener una percepción positiva del trabajo, frente a un 34\% que la señalaron como negativa. La positiva está ligada en gran parte a los significados que han construido alrededor del trabajo, mismos que mostramos a continuación:

- El primero de ellos está relacionado con el desarrollo de competencias, que básicamente implican la cuestión actitudinal. Es decir, el desarrollo de carisma y de trato para convencer al cliente para que compre sus productos; para lo cual se requieren aspectos como el esfuerzo, la atención, la amabilidad, la paciencia, el respeto, etc. A lo anterior se añaden las competencias administrativas, es decir la capacidad para llevar el registro de ventas de la tienda, tanto en lo monetario, como en lo referente a los inventarios.

○ El segundo aspecto, está representado por la percepción del trabajo ligado al comercio, como una actividad clave para la adquisición de dinero. Aunque gran parte de los jóvenes afirmaron aspirar a un negocio propio como medio de subsistencia en un futuro, también eran conscientes de lo difícil que implica llevarlo a cabo:

A mí me gustaría poner un negocio (entrevistador ¿Es fácil para un trabajador de tienda de ropa poner un negocio?) No, no... necesitas tener un contacto que te eche la mano (ayude). Yo, por ejemplo, me baso en que mi cuñada tiene un local, tengo buena relación con mi patrón, tengo amigos que me pueden decir mira aquí hay un local para que lo rentes con facilidades, a eso me baso yo, pero un trabajador común, que no tenga esa relación, no, está cabrón (difícil). Si rento (un local) tengo quien me de ropa a consignación, sin que me tenga que desfalcar... platicando con amigos en el taller o aquí en los puestos, también piensan en que un negocio es la vía, porque tú ves que de aquí sale, y sale bien para el patrón, y para los que no pudimos estudiar como yo aparte del trabajo que uno tiene, en lo que piensas, bueno, al menos yo, es en un negocio, pero como te digo si no tienes un buen contacto está muy difícil...(conversación personal, hombre de 26 años, 2015).

- El tercer aspecto se relaciona con la idea del trabajo como una actividad digna, que le dota de respeto y de reconocimiento por parte de la sociedad a la que pertenece, además que le permite desarrollarse económicamente de manera legal. 
- El cuarto aspecto positivo está constituido por el espacio de socialización que representa el lugar de trabajo. Las relaciones de amistad creadas y la posibilidad de encontrar relaciones de noviazgo, implican factores importantes que, aunque se llevan a cabo en el espacio de trabajo, trascienden a otros aspectos de la vida que son muy significativos para el joven, como lo son las relaciones afectivas y la posibilidad de construir una vida en pareja.

- El quinto aspecto considera que el trabajo es igual a salario.

- El sexto refiere a que el trabajo es el medio que permite ayudar a la familia y a su vez al consumo de bienes o servicios de interés personal. Los jóvenes referían constantemente que parte de su sueldo era para apoyar con el sustento de su familia, además del gasto que implicaba la alimentación y el transporte durante la semana de ellos mismos.

Los seis aspectos antes señalados forman parte del conjunto de significados que los jóvenes han construido del trabajo en este contexto (gráfica 2).

Gráfica 2: Significados del trabajo para los jóvenes de Moroleón y Uriangato

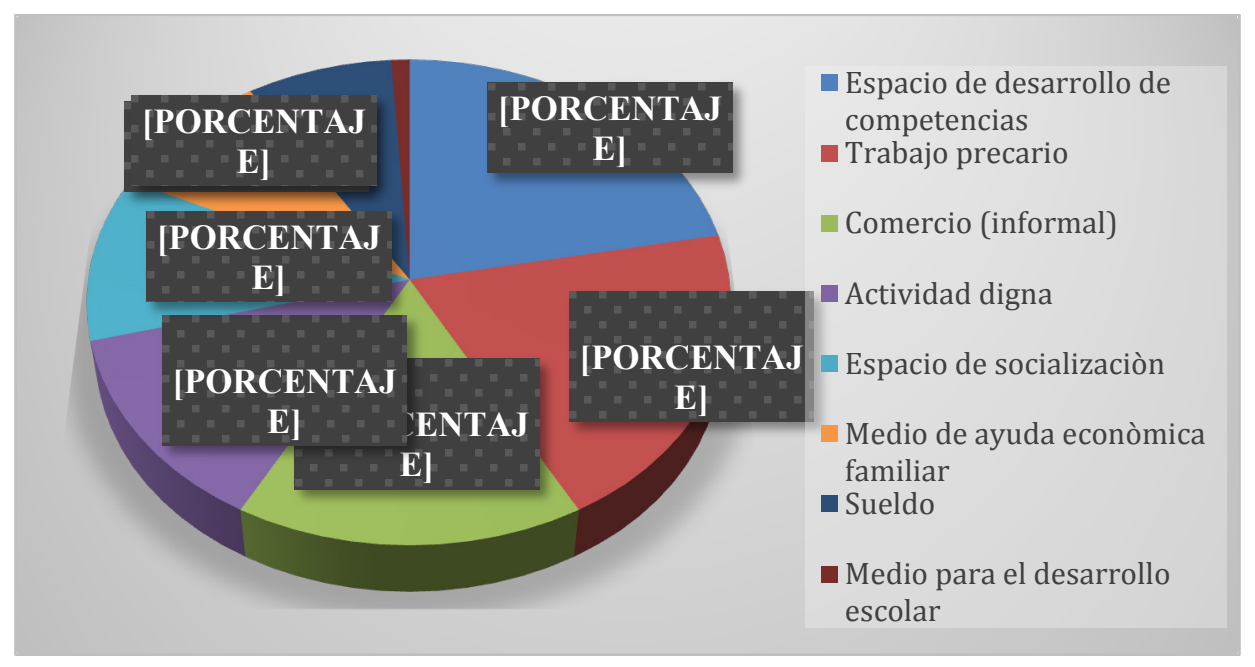

Fuente: Elaboración propia con datos de la carta asociativa aplicada en el estudio. 
A la par de los significados ya mencionados, que sustentan nuestra afirmación de la percepción positiva del trabajo, también se encuentran otros que complementan al conjunto de ideas relacionadas con el trabajo, ejemplo de ello son la idea de trabajo precario y la de que el trabajo es un medio para desarrollarse en el aspecto escolar. Si bien los jóvenes no mencionan la expresión trabajo precario, hacen referencia a situaciones como la larga jornada laboral, la carencia de prestaciones sociales, los sueldos bajos y la ausencia de contratos que establezcan una relación laboral con el patrón (Mora, 2011; Reygadas, 2011 y Rojas y Salas 2011).

En relación con la idea de que el trabajo es un medio para el desarrollo académico, se puede observar en la gráfica 3, que se presentó con muy poca frecuencia. Las y los jóvenes que referían aspiraciones de estudiar, tenían la característica de haber concluido la preparatoria y su plan era juntar dinero para después entrar a la universidad. Lo que concuerda con otros análisis que se han desarrollado en estas comunidades y en los que se observa que lo académico no es relevante y cómo reflexionamos adelante se debe analizar la relación escuela-trabajo. A pesar de que abandonar su trabajo por motivos escolares se presenta en segundo lugar, la distancia que guarda con la que se presenta en primer lugar, se explica porque al abandonar los estudios, hay mejoría en el sueldo (gráfica 3).

\section{Gráfica 3: Motivos para dejar el trabajo}

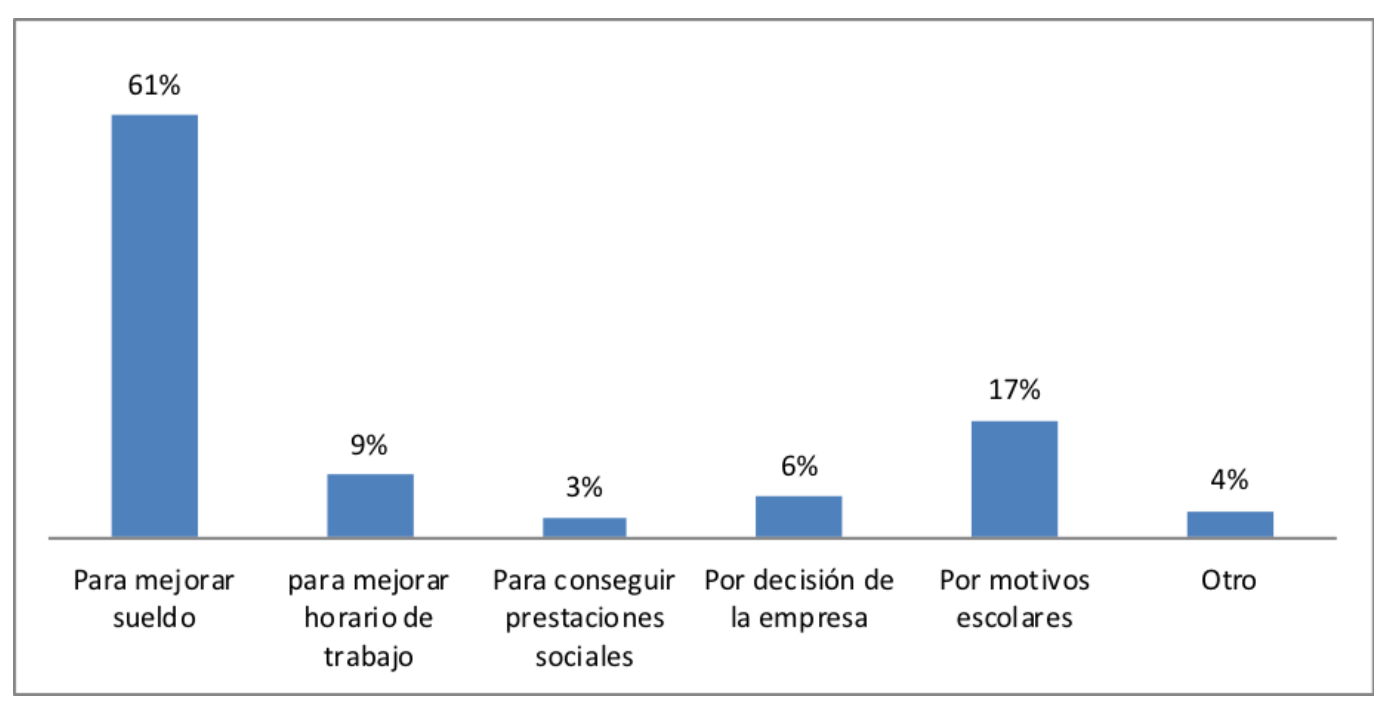


Fuente: Elaboración propia con datos del cuestionario aplicado en el estudio.

Los trabajos ofrecidos en esta ruta de comercio implican jornadas de trabajo largas que dificultan llevar a cabo tanto actividades laborales como académicas, además de la valoración que se da del trabajo por encima de la escuela:

Yo estudiaba el bachillerato aquí en Uriangato, pero después de unos problemas de salud que tuvo mi papá la dejé porque se necesitaba dinero. El Seguro Popular no alcanzaba a cubrir todo así que ni modo, pero no me arrepiento, de hecho a veces me dice mi mamá que vuelva a entrar pero ya no quiero, mira, aquí trabajo para mi tía, si ves es un puesto chico pero si sale (el ingreso es suficiente), yo gano 120 diarios y con eso me alcanza para darle a mi mamá, para ir a cenar con mi novia los domingos, que es el día más tranquilo para mí y para ella...(conversación personal, hombre de 20 años, 2016).

Los resultados encontrados refieren que los jóvenes encuentran en su espacio de trabajo la formación que ellos consideran necesaria para su progreso laboral bajo el desarrollo de competencias que en su lugar de trabajo tienen sentido y que se tornan importantes para su desenvolvimiento.

\section{Discusión y conclusiones}

De acuerdo con los resultados mostrados, la informalidad es una condición presente en la vida laboral de los jóvenes trabajadores de ambos municipios, pues entablan relaciones laborales sin contrato de trabajo, lo que propicia que los patrones sólo se responsabilicen por el sueldo del trabajador. En este sentido se configuran relaciones de trabajo, que atentan contra los derechos laborales en pos de lograr reducciones en los costos de producción, como apuntan Tokman (2011) y Maza y Gutiérrez (2015).

Las condiciones en las que los jóvenes se incorporan al mercado de trabajo son de precariedad e informalidad, combinada con los bajos ingresos, todo enmarcado por el origen social que los determina (Saraví, 2009). Con el objetivo de entender esa problemática con detalle hemos analizado la forma en la que los jóvenes conciben su propio trabajo. Utilizamos las representaciones sociales como una herramienta para captar la subjetividad.

Nos referimos a los jóvenes que son los marginados del modelo productivo que se desarrolla en Moroleón-Uriangato, aquellos que acceden a los puestos de trabajo por 
carecer de redes sociales ${ }^{8}$ que les permitan tener mejores opciones de empleo. Sin embargo, estas actividades, a pesar de ser informales y con salarios bajos, responden al primer rasgo del trabajo ideal de los jóvenes, según Pérez Islas y Urteaga (2001), que es la posibilidad de tener un negocio propio. Este factor es fundamental en la construcción de una valoración positiva del mismo, la cual se puede comprender mejor con la idea de los mecanismos de compensación (Reygadas, 2008). En este sentido, se vuelven fundamentales las redes de solidaridad, para la configuración de los nuevos negocios y para el funcionamiento del modelo de negocio. Nos referimos aquí, también, a los mecanismos de reciprocidad, mismos que permiten intercambios, se basan en las redes de ayuda mutua y que son centrales para el funcionamiento del modelo, a lo que se añade el esfuerzo individual, mismo que Reygadas (2008) señaló como resiliencia, entendida ésta como una capacidad creadora que permite afrontar las condiciones de marginación y que incluye una serie de estrategias que implican respuestas como las que podemos ver en estos tipos de trabajos, donde las empresas familiares y los negocios informales, se intercalan con el trabajo arduo, o cómo lo menciona Bourgois (2015), en un entorno de economía subterránea en donde confluyen diversas configuraciones del trabajo y estrategias informales para la sobrevivencia.

Otro aspecto que se hace patente es la desarticulación entre educación y trabajo, los datos nos muestran que la escuela no resulta una alternativa válida para los jóvenes; y que el mundo del trabajo resulta "suficiente" para ellos. De tal forma que se "plantean ciertos cuestionamientos acerca de la competencia entre ambas actividades, el valor que los propios jóvenes otorgan a una y otra en sus proyectos de vida y la forma en que esta relación subjetiva entre escuela y trabajo evoluciona con el paso del tiempo" (Saraví, 2009: 96). A lo que añadimos la condición local de no valorar la educación escolar.

La condición de informalidad bajo la cual trabajan particularmente los jóvenes que participaron en el estudio, propicia escenarios de precariedad laboral, es decir, de bajos salarios, falta de prestaciones, jornadas irregulares, largas o cortas, carentes de seguridad social, violatorios de los derechos laborales e inexistencia de negociación colectiva (Maza,

\footnotetext{
${ }^{8}$ Las redes sociales entendidas con las conexiones sociales y de amistad que pueden coadyuvar a que los jóvenes consigan un empleo con mayor facilidad.
} 
2008; Mora, 2011; Reygadas, 2011 y Rojas y Salas 2011). Sin embargo, el grupo de trabajadores que analizamos construyen sus alternativas a partir de su propia realidad, a partir de las cuales reconfiguran condiciones que podrían servir para reducir las alternativas.

Los jóvenes estudiados nunca habían entablado alguna relación laboral en condiciones de formalidad: con un contrato, ni con prestaciones sociales; por lo que ven dicha situación como algo normal $\mathrm{y}$, por tanto, no les genera mayor conflicto, pues entienden que siempre ha sido así. Esta situación se relaciona con lo comentado por Reygadas (2011) en cuanto a que la precariedad laboral en México no es una condición reciente, por el contrario, dicha situación ha estado presente por largo tiempo, lo que ha llevado al trabajador a no problematizar la situación, sino a tomar dicha condición como una propiedad misma del trabajo, por lo que se normaliza, no se cuestiona y en algunas condiciones se aprovecha.

Cabe mencionar que los conceptos de informalidad y precariedad no son sinónimos puesto que conllevan diversos componentes conceptuales que los permite distinguirse, no obstante, para el análisis de nuestros datos es importante recalcar sobre los procesos que se vive en el trabajo en estos lugares. En estos lugares, la mayoría de los negocios se encuadran en la informalidad, en tanto que no tienen vínculo con las prestaciones sociales propias de un empleo formal, sin embargo, los propietarios y trabajadores de éstos negocios informales, logran alcanzar salarios más altos en comparación de los empleados que se encuentran dentro del grupo de los asalariados formales, los cuales representan la minoría (Denham y Tilly, 2013). En estos espacios productivos se observan componentes de precariedad, en tanto que existen empleos sin contratos, los salarios son bajos, las jornadas son extensas y parciales, no existen acuerdos en materia de negociación colectiva, entre otras características (Reygadas, 2011). Todos estos aspectos son negativos si la mirada se hace desde una perspectiva teórica de la formalidad laboral, sin embargo, desde la percepción de los sujetos, estas condiciones no parecen afectar la economía y las dinámicas laborales en el lugar, por el contario, favorecen en algunos procesos de la producción y en las relaciones socio-laborales. 
Esto no implica que sea una situación legalmente correcta, ni proclive para que se dé una movilidad social sustanciosa, pues como lo comenta el propio Reygadas (2011) la precarización del trabajo es un fenómeno que se ha potencializado con mayor fuerza a partir de las transformaciones laborales emanadas tras la década de 1980 y que ha recrudecido las condiciones laborales, las cuales ya eran de por si malas. Dicha situación repercute en los altos índices de pobreza y de exclusión social que se viven en México y, particularmente, en en el estado de Guanajuato, como lo apunta CONEVAL (2016).

Si bien las condiciones de precariedad se han presentado en muchos textos y se han analizado de diversas perspectivas, creemos que la aportación de este trabajo radica en mostrar la forma en la que los jóvenes construyen sus alternativas en condiciones de marginación. De tal forma que no permanecen pasivos y formulan soluciones a las necesidades cotidianas que tienen que ver con los rubros de salud, vivienda o ingresos económicos. Han encontrado en su propia condición de juventud, en instituciones como el Seguro Popular y en su capital social (familiares o amistades) formas alternas para solucionar problemas en los ámbitos antes mencionados.

A partir de las representaciones sociales sobre el trabajo que se caracterizan por estar configuradas por elementos positivos, que dejan ver la valoración que se tiene del trabajo, a pesar de las condiciones en las que se realiza. De manera preponderante encontramos las competencias dirigidas al saber hacer, es decir, para el desarrollo de habilidades que les permitan resolver problemas de su vida cotidiana en su lugar de trabajo. Desde la perspectiva del joven trabajador de las tiendas de ropa en Moroleón y Uriangato, su trabajo significa, entre otras cosas, un espacio de formación donde desarrolla aspectos actitudinales y habilidades administrativas que los aplican para seguir ejerciendo su labor, o bien, para en un futuro pasar de empleados a dueños de su propio negocio. Además de que el incremento de habilidades les garantiza hacerse independientes en su trabajo.

A lo anterior debemos añadir los significados que han construido en torno al ingreso económico, que simboliza acceso a bienes y servicios básicos, como lo son alimentación, salud y gastos de vivienda. Por otra parte, la asociación del trabajo a escenarios de socialización y de capital social, surge como elemento importante en los significados que 
tienen los jóvenes sobre el trabajo, pues se convierten en espacios donde las relaciones sociales pueden convertirse en alternativas de crecimiento laboral o de ampliación de los proyectos económicos a futuro.

La conexión de trabajo con el comercio, como otro significado erigido por los jóvenes, permite dimensionar la importancia del contexto social y cultural en la construcción de representaciones sociales (Moscovici, 1988; Jodelet, 1988 y Abric, 2001) pues, como se comentaba en apartados anteriores, Moroleón y Uriangato se caracterizan por una tradición comercial que rebasa más de un siglo, en el que se desarrolló un mercado que ha sobrevivido a crisis económicas y que ha encontrado en la informalidad, en la diversificación de su producción y comercialización de ropa, las principales alternativas de crecimiento. El significado del trabajo relacionado con el comercio concede al joven pensarse con alternativas de trabajo dentro de este rubro, lo que según sus experiencias les permitiría, siendo dueños de algún negocio, conseguir su propio dinero, ser dueños de su tiempo y tener jornadas de trabajo menos pesadas.

Finalmente, en su totalidad, los significados que los jóvenes construyen sobre el trabajo en el contexto antes descrito permitirían vislumbrar la reproducción de la informalidad y de las condiciones de trabajo precario, con los matices ideológicos que se implican en la construcción de un futuro viable en mejores condiciones, situación que aún parece viable en este modelo productivo. Donde sigue siendo relevante el pensar en la cohesión social como factor que favorece mejores condiciones para los jóvenes trabajadores.

\section{REFERENCIAS}

ABRIC, Jean C. Prácticas sociales y representaciones. México, D.F., Ediciones Coyoacán, 2001.

ARIAS, Patricia. Nueva rusticidad mexicana. México, D.F., Conaculta, 1992. 
BOURGOIS, Philippe. En busca de respeto. Vendiendo crack en harlem. Siglo XXI Editores, 2015, págs. 426.

CONASAMI. Comisión Nacional de los Salarios Mínimos. 2018. Disponible en: https://www.gob.mx/conasami

CONEVAL. Consejo Nacional de Evaluación de la Política de Desarrollo Social: Medición de pobreza, Estados Unidos Mexicanos. 2016. Recuperado de https://www.coneval.org.mx/Medicion/Paginas/PobrezaInicio.aspx

DENHAM, Diana., y TILLY, Chris. Sueños de éxito, realidades estancadas: Aspiraciones y movilidad en el comercio informal y formal en México. En Revista Latino-americana de Estudos do Trabalho, 18(30), 2013, 9-42.

DE LA GARZA, Enrique, \& NEFFA, Julio C. Trabajo y modelos productivos en América Latina: Argentina, Brasil, Colombia, México y Venezuela luego de la crisis del modo de desarrollo neoliberal. Buenos Aires, Clacso, 2010.

DE LA GARZA, Enrique. Las teorías sobre la reestructuración productiva y América Latina, en: DE LA GARZA, Enrique (coord.), Tratado Latinoamericano de Sociología del Trabajo, México, D.F., Fondo de Cultura Económica. 2000. Pp. 716-734.

DENUE. Directorio Estadístico Nacional de Unidades Económicas. Instituto Nacional de Estadística y Geografía, 2018. Recuperado de http://www.beta.inegi.org.mx/app/mapa/denue/default.aspx

ENOE (2014). Tasas de informalidad trimestral 2005-2014. Instituto Nacional de Estadística y Geografía. 2014. Recuperado de http://www3.inegi.org.mx/sistemas/tabuladoresbásicos/tabtema.aspx?s=est\&c=33619

GARCIA, Alejandro. El capital social individual en la industria y el comercio de ropa en Uriangato, Guanajuato. Una aproximación desde el análisis de redes personales, en: MAZA, Octavio y ORTIZ, Dolly (coord.), En el mismo barco. Reflexiones sobre la producción, el trabajo y las redes sociales: El caso de Uriangato, Guanajuato, Guanajuato, México, Editorial de la Universidad Autónoma de Aguascalientes. 2015. Pp. 132-195. 
INEGI. Conteo de Población y vivienda. Instituto Nacional de Estadística y Geografía. 2010. Recuperado de http://www.inegi.gob.mx.

INEGI. Encuesta Intercensal. Instituto Nacional de Estadística y Geografía. 2015. Recuperado de: http:/www.beta.inegi.org.mx/proyectos/enchogares/especiales/intercensal/

JAMES, Clifford. The predicament of culture. Cambridge, Harvart University Press, 1988.

JODELET, Denise. La representación social: fenómenos, conceptos y teoría, en: MOSCOVICI, Serge, Psicología social, II: Pensamiento y vida social. Psicología social y problemas sociales, España, Paidós. 1988. Pp. 469-494.

MAZA, Octavio M. y GUTIERREZ, Edgar. Modelos productivos y distritos industriales. En MAZA, Octavio, y ORTIZ, Dolly (coord.), En el mismo barco. Reflexiones sobre la producción, el trabajo y las redes sociales: El caso de Uriangato, Guanajuato. Guanajuato, México, Editorial de la Universidad Autónoma de Aguascalientes. 2015. Pp. 94-132.

MAZA, Octavio. Alternativas analíticas de las teorías de mercados de trabajo, para el estudio de las formas precarias de ocupación. En: LOPEZ, Rosalía, RAYA, Rogelio y RUIZ, Jesús (coord.), Educación, ejercicio profesional y mercados de trabajo México, CEIICH-UNAM; U de G; UAA e IMCED. 2008. Pp. 227-243.

MORA, Minor (2011). El empleo precario asalariado y globalización: enseñanzas desde Costa Rica, en: PACHECO, Edith, DE LA GARZA, Enrique, y REYGADAS, Luis (coord.), Trabajos atípicos y precarización del empleo, México: Colegio de México. 2011. Pp. 161-198.

MOSCOVICI, Serge., ABRIC, J-C., IBÁÑEZ, Tomás, y ROSENBAUM, David. Psicología Social I. Influencia y cambio de actitudes. Individuos y grupos. España, Paidós, 1988.

NEGRETE, Rodrigo. El concepto estadístico de informalidad y su integración bajo el esquema del Grupo Delhi. Revista Internacional de Estadística y Geografía Realidad, Datos y Espacio, vol. n. 3, 2011, pp. 76-95. Recuperado de 
https://www.inegi.org.mx/rde/2011/09/04/el-concepto-estadistico-de-informalidad-y-suintegracion-bajo-el-esquema-del-grupo-de-delhi/

PASILLAS, Omar. Incertidumbres y estrategias de subsistencia familiares. Un estudio sobre la experiencia del desempleo en dos regiones productivas: Salto de Tepuxtepec, Michoacán y Moroleón-Uriangato, Guanajuato (Tesis de Maestría). Querétaro, Qro. México. Facultad de Filosofía, Universidad Autónoma de Querétaro, México, 2016.

PEREZ, José A, y URTEAGA, Maritza. Los nuevos guerreros del mercado. Trayectorias laborales de jóvenes buscadores de empleo, en PIECK, Enrique, (coord.), Los jóvenes y el trabajo. La educación frente a la exclusión social. México, Universidad Iberoamericana. 2001. Pp. 355-399.

REYGADAS, Luis. Introducción: Trabajos atípicos, trabajos precarios: ¿Dos caras de la misma moneda?, en PACHECO, Edith., DE LA GARZA, Enrique y REYGADAS, Luis (coord.), Trabajos atípicos y precarización del empleo. México, Colegio de México. 2011. Pp. 21-43.

REYGADAS, Luis. La apropiación. Destejiendo las redes de la desigualdad. Barcelona, Anthropos, 2008.

ROJAS, Georgina, y SALAS Carlos. Precariedad Laboral y la estructura del empleo en México, 1995-2004, en PACHECO. Edith, DE LA GARZA, Enrique y REYGADAS, Luis, (coord.), Trabajos atípicos y precarización del empleo. México, Colegio de México. 2011. P.p. 117-154.

SALAS, Carlos. El Sector informal: auxilio u obstáculo para el conocimiento de la realidad social en América Latina, en DE LA GARZA, Enrique E. (coord.), Teorías sociales y estudios del trabajo: nuevos enfoques, España, Anthropos. 2006. Pp. 130-148.

SARAVÍ, Gonzalo. Desigualdad en las experiencias y sentidos de la transición escuelatrabajo. Papeles de Población, vol.15, no. 59, pp. 83-118, 2009. Disponible en: https://rppoblacion.uaemex.mx/about 
SARAVÍ, Gonzalo. Juventudes fragmentadas socialización, Clase y Cultura en la construcción de la desigualdad. México, Facultad Latinoamericana de Ciencias Sociales, Sede México, 2015.

SEIEG. Sistema Estatal de Información Estadística y Geográfica e Instituto de Planeación, Estadística y Geografía. 2014. Cartografía Guanajuato, Mapas temáticos sobre Guanajuato. Disponible en:

http://seieg.iplaneg.net/seieg/doc/mapas fb/Zonas\%20Metropolitanas\%20de\%20Guanajuat o/

SIERRA, Julieta. (2003). Las familias micro empresariales de la industria del vestido en Moroleón y Uriangato, Guanajuato. (Tesis de Maestría). México, D.F.; Centro de Investigación y Estudios Superiores en Antropología social (CIESAS).

TOKMAN, Víctor. Informalidad en América Latina: Balance y perspectivas de políticas. Revista Internacional de Estadística y Geografía. Realidad, Datos y Espacio, vol. 2, no. 3, pp. 16-31. 2011.

VANGSTRUP, Ulrik. Moroleón: La pequeña ciudad de la gran Industria. ESPIRAL, Estudios sobre Estado y Sociedad, vol. 2, no. 4, pp. 101-134, septiembre-diciembre, 1995

ZAPATA, Francisco. Tiempos neoliberales en México. México, Colegio de México, 2005.

Recebido 19/03/2020

Aprovado 10/04/2020 